\title{
miR-939-3p promotes epithelial-mesenchymal transition and may be used as a prognostic marker in hepatocellular carcinoma
}

\author{
FEI CHEN ${ }^{1}$, XIAOYING NI ${ }^{1}$, LINGXIU CHEN $^{1}$, XIAOYAN WANG $^{1}$ and $\mathrm{JI} \mathrm{XU}^{2-4}$ \\ ${ }^{1}$ Department of Digestive Center, Tiantai Branch of Zhejiang Provincial Peoples' Hospital, Taizhou, Zhejiang 317200; \\ ${ }^{2}$ Department of Gastrointestinal and Pancreatic Surgery, Zhejiang Provincial Peoples' Hospital; \\ ${ }^{3}$ Key Laboratory of Gastroenterology of Zhejiang; ${ }^{4}$ School of Clinical Medicine, \\ Peoples' Hospital of Hangzhou Medical College, Hangzhou, Zhejiang 310000, P.R. China
}

Received April 16, 2019; Accepted December 13, 2019

DOI: $10.3892 / 01.2020 .11361$

\begin{abstract}
Hepatocellular carcinoma (HCC) is one of the most common types of cancer worldwide with a high morbidity and mortality rate. An increasing number of studies have demonstrated that microRNAs (miRNAs) serve an important role in HCC. The present study investigated the role of miR-939-3p in HCC. It was demonstrated that miR-939-3p was upregulated in HCC cell lines and HCC tissues compared with normal liver cell lines and paired normal tissues, respectively. It was also found that upregulation of miR-939-3p expression levels in HCC tissues was associated with a less favorable prognosis. Moreover, the overexpression of miR-939-3p in LM3 cells enhanced the metastatic capacity of these cells and promoted epithelial-mesenchymal transition (EMT). In contrast, miR-939-3p inhibition decreased the invasive capacity of HCC cells and EMT. Potential binding target of miR-939-3p to estrogen receptor 1 (ESR1) were predicted using TargetScan. The expression levels of miR-939-3p were negatively associated with ESR1 in HCC tissues based on data from The Cancer Genome Atlas. A luciferase reporter assay was used to confirm ESR1 as a direct downstream target of miR-393-3p. The miR-939-3p/ESR1 axis may be a potential novel target for the treatment of HCC.
\end{abstract}

\section{Introduction}

Hepatocellular carcinoma (HCC) is a type of primary liver cancer and is one of the most common malignant types of cancer worldwide, with high morbidity and cancer associated mortality rates (1). The incidence rate of HCC in China is the highest in the world due to an increased rate of hepatitis B virus infection (2). Furthermore, the overall survival rate has remained unsatisfactory for the last decade at 22-35\% (3). Although numerous studies

Correspondence to: $\mathrm{Dr} \mathrm{Ji} \mathrm{Xu}$, Department of Gastrointestinal and Pancreatic Surgery, Zhejiang Provincial Peoples' Hospital, 158 Shangtang Road, Hangzhou, Zhejiang 310000, P.R. China

E-mail: xuji120@163.com

Key words: hepatocellular carcinoma, microRNA-939-3p, estrogen receptor 1 have been performed, the carcinogenesis and progression of HCC remains unclear (4-6). Therefore, identifying and clarifying the molecular mechanisms involved in development and progression of HCC may improve prognostic outcomes.

It has been reported that microRNAs (miRNAs/miRs), which are highly conserved, small non-coding RNAs, 19-25 nucleotides in length and abundantly expressed in animals $(7,8)$, may bind to the 3'-untranslated region (UTR) of target genes and inhibit the expression of these genes through post-transcriptional regulation of mRNAs (9). A number of studies have demonstrated that miRs, including miR-21, miR-197-3p and miR-497-5p, serve an important role in apoptosis, cell proliferation, differentiation and metastasis (10-13). A previous study reported that inhibition of miR-939-3p may suppress the development of human non-small cell lung cancer (NSCLC) via the upregulation of metalloproteinase 2 (14). However, to the best of our knowledge, the function of miR-939-3p in HCC remains unknown.

Estrogen receptor 1 (ESR1), a ligand-activated transcription factor, may directly bind to the transcription factor (TF) complex and lead to altered functions of proteins in the cytoplasm, for examples activation of eNOS or regulation of gene expression through phosphorylation (15). Numerous studies have demonstrated that ESR1 acts as a tumor suppressor in various cancer types. Yang et al (16) reported that ESR1 directly regulates the hypoxia-inducible factor 1 or the pathway associated with the anti-estrogen response in breast cancer. An ESR $\alpha$ inhibitor activated the unfolded protein response, blocked protein synthesis and induced tumor regression in HCC (17). Hishida et al (18) predicted that ESR1 is a tumor suppressor gene in HCC by triple-combination array analysis. Additionally, Tu et al (19) demonstrated that ESR1 overexpression mediated apoptosis in Hep3B cells by binding with SP1 proteins. However, to the best of our knowledge, the effect of ESR1 on the metastasis of HCC cells has not been studied. Therefore, the aim of the present study was to determine the potential gene binding of miR-939-3p and the function of miR-939-3p in HCC.

\section{Materials and methods}

Tissue samples. The present study was approved by The Institutional Ethics Committee of Zhejiang Provincial People's Hospital (Hangzhou, China). The clinical data were obtained 
from The Cancer Genome Atlas (TCGA, portal.gdc.cancer. gov/).

Cell culture. The HCC cell line (HCCLM3) was obtained from the American Type Culture Collection. Cells were cultured at $37^{\circ} \mathrm{C}$ with $5 \% \mathrm{CO}_{2}$ in Minimum Essential Medium (MEM; Thermo Fisher Scientific, Inc.) containing 10\% FBS (Thermo Fisher Scientific, Inc.). This cell line was authenticated by short tandem repeats profiling.

Reverse transcription-quantitative (RT-q)PCR. Total RNA was extracted using TRIzol ${ }^{\circledR}$ reagent (Invitrogen; Thermo Fisher Scientific, Inc.). RNA was then reverse transcribed to cDNA using PrimeScript ${ }^{\mathrm{TM}}$ RT Master mix (cat. no. RR036A; Takara Bio, Inc.), according to the manufacturer's protocol. qPCR was performed using an ABI 7500 (Thermo Fisher Scientific, Inc.). The primer sequences were as follows: miR-939 forward, 5'-TGGGGAGCTGAGGCTCTG-3' and reverse, 3'-AGTGCAGGGTCCGAGGTATT-5'; U6 forward, 5'-CTCGCTTCGGCAGCACA-3' and reverse, 3'-AACGCT TCACGAATTTGCGT-5'; and ESR1 forward, 5'-CCGGCT CCGTAAATGCTACG-3' and reverse, 3'-TCCAGCAGACCC CACTTCAC-5'. U6 was used as the internal control.

Transfection. miR-939-3p mimic, miR-939-3p inhibitor and ESR1 small interfering (si) RNA were obtained from Shanghai GenePharma Co., Ltd. Cells were seeded in 6-well plates $\left(3 \times 10^{5} /\right.$ well) and cultured for $24 \mathrm{~h}$ before transfection. Lipofectamine $^{\circledR} 3000$ (Invitrogen; Thermo Fisher Scientific, Inc.) was used for transfection, according to the manufacturer's protocol. The sequences of the miRNAs were as follows: hsa-miR-939 mimic sense, UGGGGAGCUGAGGCUCUG GGGGUG and antisense, CCCCCAGAGCCUCAGCUCCCC AUU; mimics negative control (NC) sense, UUCUCCGAACGU GUCACGUTT and antisense, ACGUGACACGUUCGGAGA ATT; and hsa-miR-939 inhibitor, CACCCCCAGAGCCUCAGC UCCCCA; and inhibitor NC, CAGUACUUUUGUGUAGUA CAA. The sequences of the ESR1 siRNA were as follows: Sense, GCAAGUUGAUCUUAGUUAAGU and antisense, UUAACU AAGAUCAACUUGCUG; siRNA NC (cat. no. siN05815122147; Guangzhou RiboBio Co., Ltd.,) was used as the siRNA negative control, but the sequence was not provided by the supplier.

Western blot. Tissues or cells were lysed with RIPA lysis buffer (Thermo Fisher Scientific, Inc.) containing 1\% protease inhibitor cocktail. The concentration of the extracted protein was analyzed using BCA kit (Beyotime Institute of Biotechnology). Microplate reader and Gen5 software version 2 (BioTek Instruments, Inc) were used to detect the quantification of protein expression.12\% SDS-PAGE was used to resolve the proteins, which were then transferred to PVDF membranes. PVDF membranes were blocked with 5\% fat-free milk at room temperature for $2 \mathrm{~h}$, followed by incubation with primary antibodies (all 1:1,000) against ESR1 (cat. no. MA5-145011; Invitrogen; Thermo Fisher Scientific, Inc.), matrix metalloproteinase (MMP) 2 (cat. no. MA5-14186), MMP9 (cat. no. MA5-15886), vimentin (cat. no. MA5-11883) and GAPDH (cat. no. AM4300; all from Invitrogen; Thermo Fisher Scientific, Inc.) at $4^{\circ} \mathrm{C}$ overnight. Subsequently, the PVDF membranes were incubated with the secondary antibody $(1: 2,000$; cat. no. ab6721; Abcam) for $1 \mathrm{~h}$ at room temperature. Signals were visualized using ECL substrate (Pierce; Thermo Fisher Scientific, Inc.).

Dual-luciferase reporter assay. PmirGLO plasmids containing the wild-type (Wt) or mutant (Mut) 3'UTR of ESR1 were purchased from Shanghai GenePharma Co., Ltd. PmirGLO plasmids were transfected into LM3 cells with miR-939-3p mimic or inhibitor with Lipofectamine ${ }^{\circledR} 2000$ reagent (Thermo Fisher Scientific, Inc.). Cells were cultured for $48 \mathrm{~h}$ prior to measurement of luciferase intensity. At $48 \mathrm{~h}$ post-transfection, the cells were lysed using radioimmunoprecipitation assay buffer (cat. no. P0013C; Beyotime Institute of Biotechnology) according to the manufacturer's protocol. A F-4500 fluorescence spectrophotometer (Hitachi, Ltd.) was used to measure the luciferase intensity according to the manufacturer's protocol and normalized to that of Renilla luciferase.

Migration and invasion assays. Cell migration and invasion ability was evaluated using a Transwell assay. LM3 cells $\left(5 \times 10^{4}\right)$ were seeded in the upper chamber with FBS-free MEM and the lower chamber contained MEM supplemented with $10 \%$ FBS. For invasion assays, membranes were coated with $50 \mu \mathrm{l}$ growth factor-reduced Matrigel (BD Biosciences). Cell migration and invasion were measured after incubation for $24 \mathrm{~h}$. Cells were stained with crystal violet dye solution for $5 \mathrm{~min}$ at room temperature, and the number of cells were counted in five randomly selected fields with a light inverted microscope at x200 magnification. Each experiment was repeated three times.

Bioinformation analysis. We predict the target gene of miRNA with TargetScan (version 5.0; genes.mit.edu/targetscan). The level of miR-939-3p in the adjacent normal tissues and HCC tissues and the Kaplan-Meier survival curve analysis in HCC patients in The Cancer Genome Atlas (TCGA) were analyzed with miRpower (kmplot.com/analysis/) (20).

Statistical analysis. Data are presented as the mean \pm standard deviation unless otherwise shown. Statistical analysis was performed using SPSS 19.0 (IBM, Corp.). Significance between groups was analyzed using an unpaired Student's t-test. The correlation between miR-939-3p and ESR1 expression levels was examined using a Pearson's correlation coefficient. The $\log$ rank test was used for survival analysis. $\mathrm{P}<0.05$ was considered to indicate a statistically significant difference.

\section{Results}

miR-939-3p is upregulated in HCC tissues and is associated with prognosis. miR-939-3p was upregulated in HCC tissues compared with paired normal tissues based on data obtained from The Cancer Genome Atlas (TCGA; $\mathrm{P}<0.05$; Fig. 1A). Furthermore, patients with upregulated expression levels of miR-939-3p exhibited significantly improved overall survival compared with patients with low expression levels of miR-939-3p in two different datasets (kmplot.com/analysis/index.php?p=background) (Fig. 1B).

miR-939-3p regulates migration, invasion and EMT of LM3 cells. To investigate the function of miR-939-3p, miR-939-3p mimics and inhibitors were used to increase or decrease the 

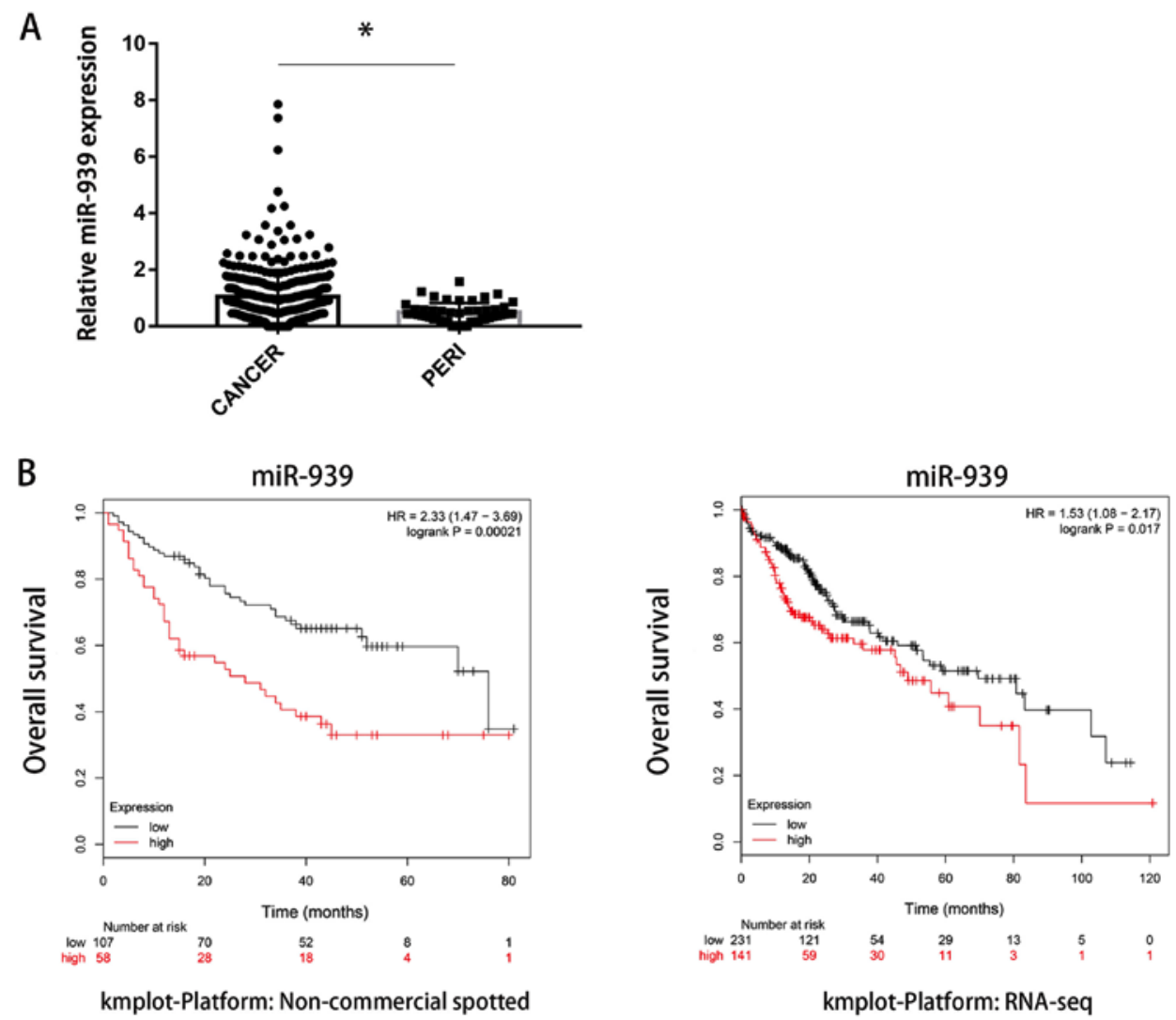

Figure 1. miR-939-3p expression is upregulated in HCC tissues. (A) miR-939-3p expression levels were significantly higher in HCC tissues compared with paired normal tissues. (B) Low miR-939-3p expression levels were associated with improved overall survival in two different datasets obtained from The Cancer Genome Atlas. "P<0.05 vs. normal tissues. HCC, hepatocellular carcinoma; miR, microRNA; PERI, peri-tumoral tissue; HR, Hazard ratio.

A
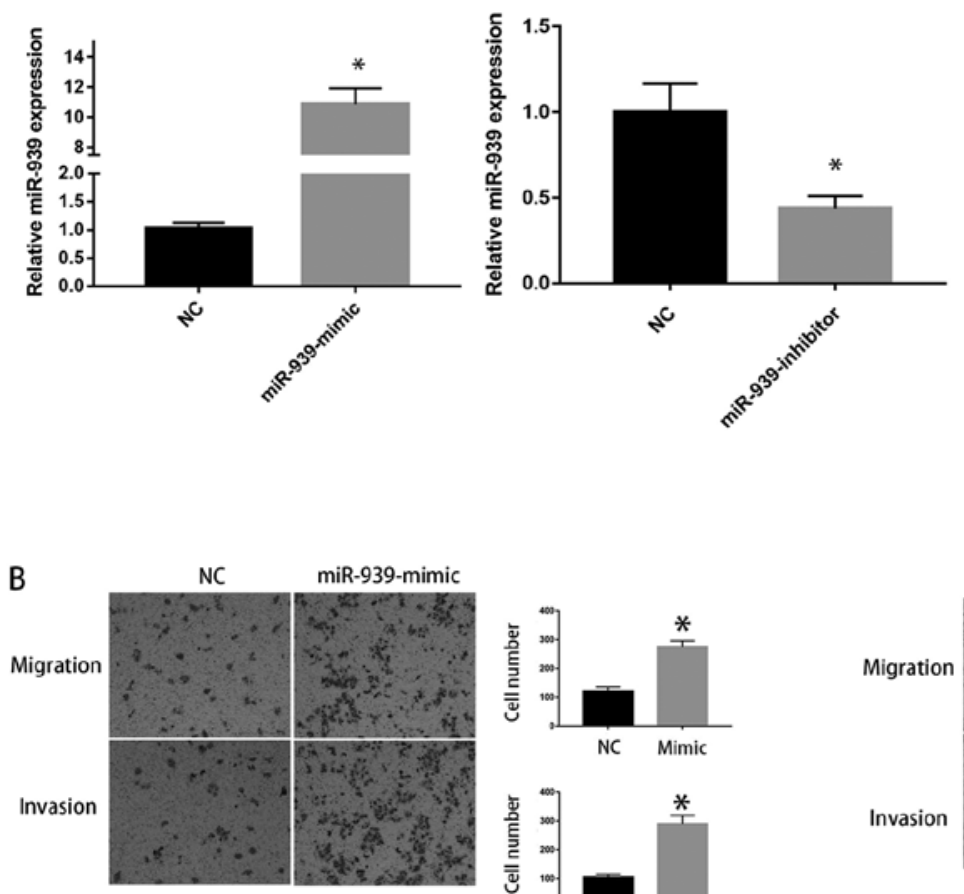

C
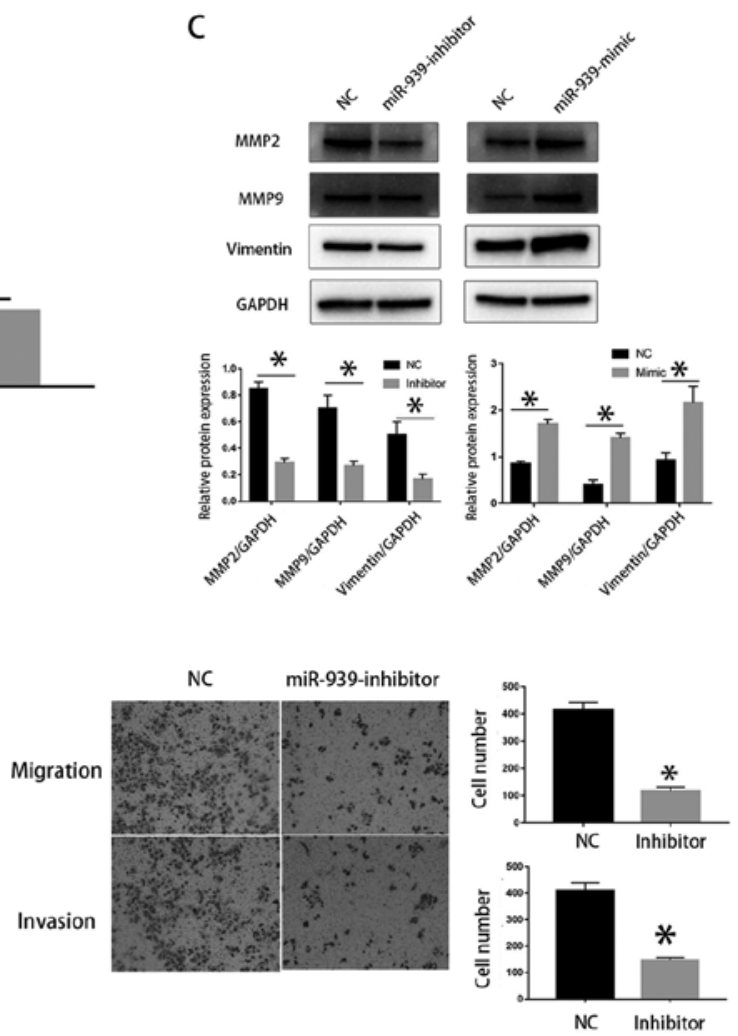

Figure 2. miR-939-3p promotes invasion and EMT in a HCC cell line. (A) Transfection efficiency of miR-939-3p mimic and inhibitor was detected by reverse transcription quantitative PCR. "P $<0.05$ vs. NC. (B) Transwell assay were performed to assess the migration of LM3 cells treated with miR-939-mimic or miR-939-inhibitor. "P<0.05 vs. NC. (C) Western blotting was used to examine the effect of miR-939-3p on EMT in LM3 cells. miR, microRNA; HCC, hepatocellular carcinoma; EMT, epithelial-mesenchymal transition; NC, negative control. 
A

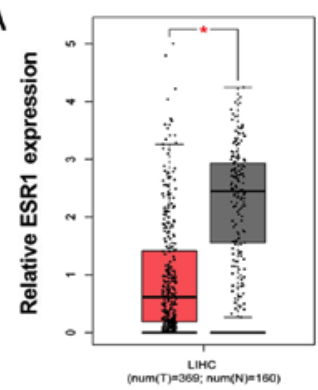

C

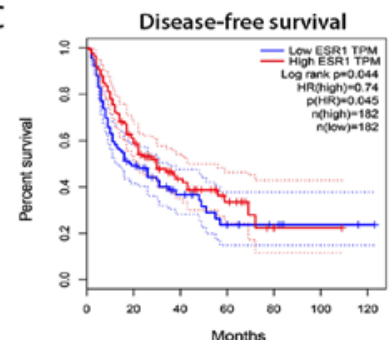

D

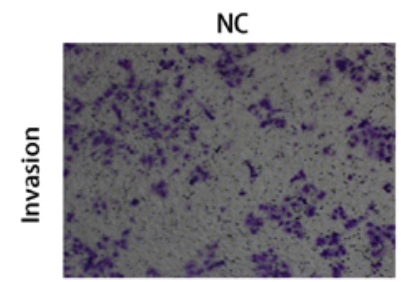

B
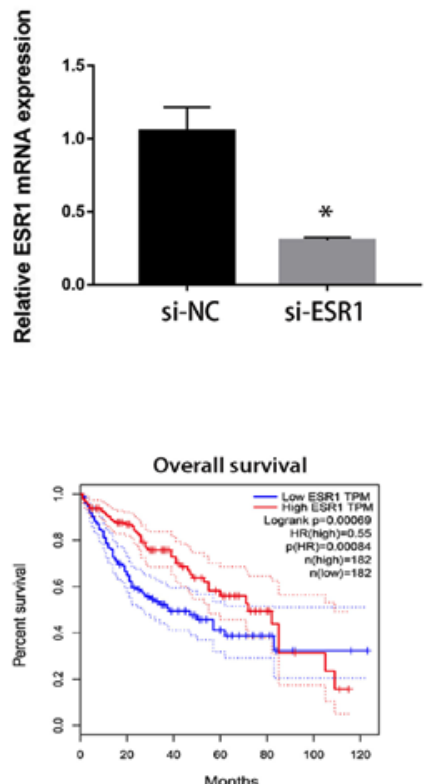

Si-ESR1

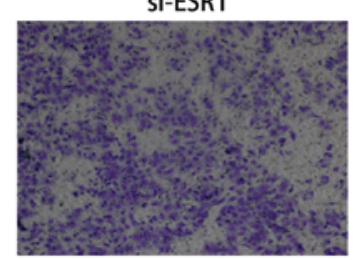

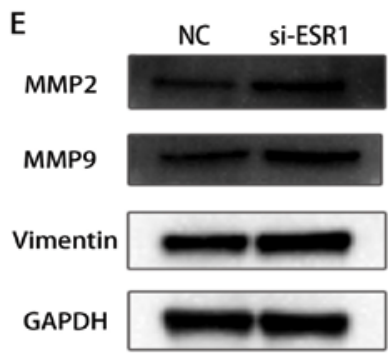
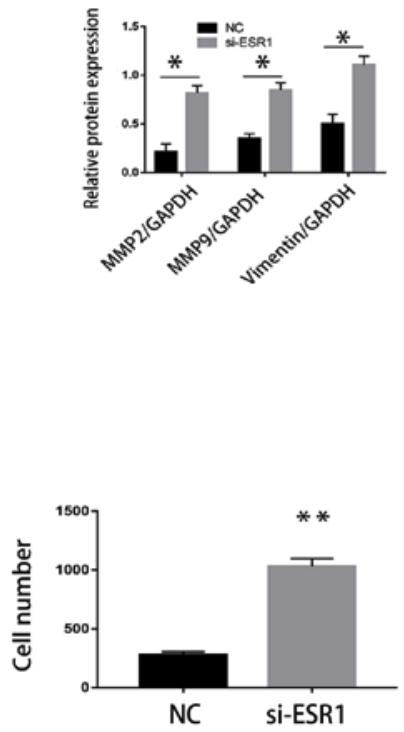

Figure 3. ESR1 is downregulated in HCC tissues. (A) ESR1 mRNA expression levels in HCC tissues and paired normal tissues. "P<0.05 vs. paired normal tissues. (B) mRNA expression levels of ESR1 in LM3 cells transfected with siRNA-ESR1 and LM3 cells transfected with siRNA-NC. "P<0.05 vs. siRNA-NC. (C) Increased ESR1 expression levels were correlated with improved disease-free survival. (D) ESR1-knockdown enhanced the migration of LM3 cells. (E) Western blotting was used to evaluate the effect of ESR1 on EMT in LM3 cells. ${ }^{* *} \mathrm{P}<0.01$ vs. NC. ESR1, Estrogen receptor 1; HCC, hepatocellular carcinoma; NC, negative control; si, small interfering; T, Tumor tissue; N, Normal tissue; LIHC, Liver hepatocellular carcinoma; HR, Hazard ratio; MMP, matrix metalloproteinase.

expression levels of miR-939-3p, respectively (Fig. 2A). LM3 cells transfected with miR-939-3p mimic exhibited an increased migratory and invasive capacity $(\mathrm{P}<0.05$; Fig. $2 \mathrm{~B})$. In addition, western blot analysis demonstrated that overexpression of miR-939-3p significantly upregulated the protein expression levels of MMP2, MMP9 and vimentin (Fig. 2C). Knockdown of miR-939-3p resulted in a reduction of invasion and expression of EMT-associated proteins in LM3 cells (Fig. 2C).

ESR1 is downregulated in HCC tissues and cell lines, and is associated with a less favorable prognosis. ESR1 expression was downregulated in HCC tissues compared with paired normal tissues based on data obtained from TCGA $(\mathrm{P}<0.05$; Fig. 3A). Furthermore, patients with lower expression levels of ESR1 exhibited improved overall survival and disease-free survival compared with patients who exhibited increased ESR1 expression levels in TCGA dataset ( $\mathrm{P}<0.05$; Fig. 3C).

ESR1 regulates the invasion and EMT of LM3 cells. The mRNA expression levels of ESR1 were lower in LM3 cells transfected with siRNA-ESR1 compared with LM3 cells transfected with siRNA-NC ( $\mathrm{P}<0.05$; Fig. 3B). It was demonstrated that ESR1-knockdown significantly increased the invasion of HCCLM3 cells ( $\mathrm{P}<0.01$; Fig. 3D). Additionally, western blotting revealed that ESR1-knockdown increased the protein expression levels of MMP2, MMP9 and vimentin in HCCLM3 cells $(\mathrm{P}<0.05$; Fig. $3 \mathrm{E})$.

miR-939-3p directly targets ESRI and regulates ESR1 expression levels in HCC cells. Binding of the ESR1 3'UTR with miR-939-3p was predicted using TargetScan. The Wt and Mut sequences were constructed and inserted into a PmirGLO vector (Fig. 4A). A significantly negative correlation between miR-939-3p and ESR1 was observed in the TCGA dataset (Fig. 4B). A dual-luciferase report assay was performed to detect the effect of miR-939-3p on ESR1 promoter activity. Decreased luciferase activity was observed in the Wt group, whereas no changes were detected in the Mut group compared with the NC group ( $\mathrm{P}<0.05$; Fig. 4C). Western blotting demonstrated that the protein expression levels of ESR1 were downregulated following transfection with miR-939-3p mimic in HCCLM3 cells (Fig. 4D), whereas, the reverse was observed following the transfection of miR-939-3p inhibitor in HCCLM3 cells (Fig. 4D).

\section{Discussion}

$\mathrm{HCC}$ is one of the most common tumor types worldwide, with high morbidity and mortality rates (1). Although numerous oncogenes and tumor suppressors have been reported in HCC (21-24), the underlying mechanisms of development and 
A

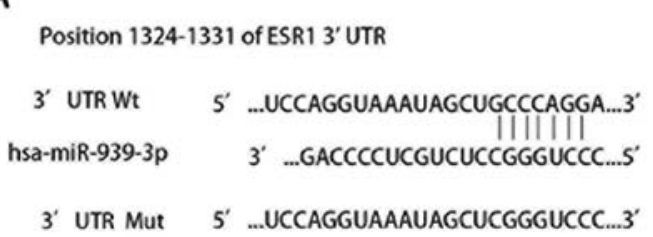

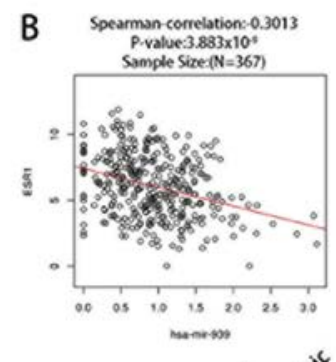

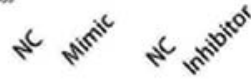

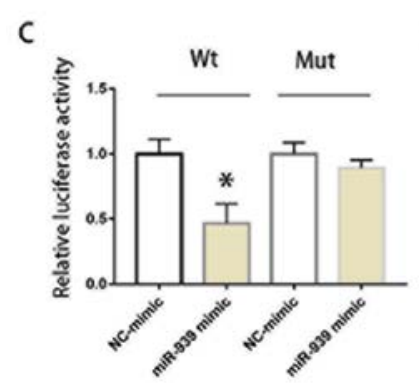
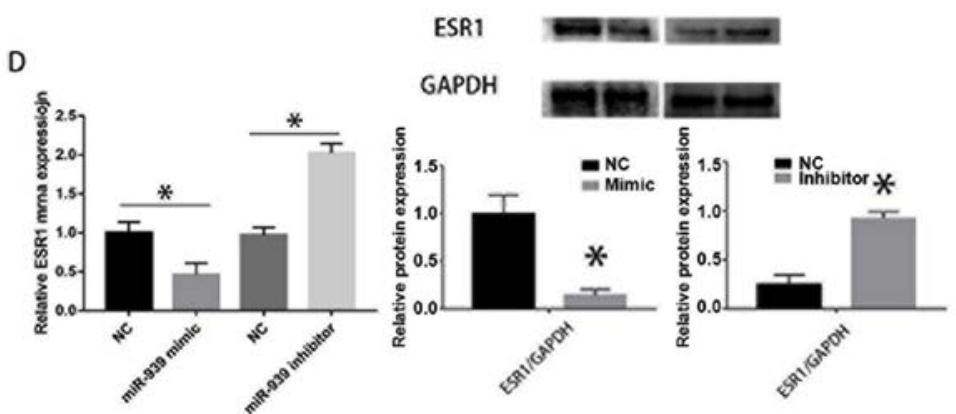

Figure 4. miR-939-3p directly binds to the 3'UTR of ESR1. (A) Wt and Mut sequences of the ESR1 3'UTR and the binding sequences of miR-939-3p. (B) Correlation of ESR1 and miR-939-3p in The Cancer Genome Atlas. (C) miR-939-3p suppressed the luciferase activity of the Wt ESR1 3'UTR, whereas the Mut miR-939-3p sequence did not in LM3 cells. "P<0.05 vs. NC-mimic (D) mRNA and protein expression levels of ESR1 were reduced following transfection with miR-939-3p mimic, whereas miR-939-3p inhibitor increased ESR1 expression in LM3 cells. *P<0.05 vs. NC. ESR1, Estrogen receptor 1; Wt, wild type; Mut, mutant; NC, negative control; UTR, untranslated region; miR, microRNA.

recurrence of $\mathrm{HCC}$ remain unclear. Over the past decade, the overall survival rate of HCC has remained unsatisfactory and is only 22-35\%. A number of genes such as CAV1, SPOCK1 and PRMT1 (25-27) may contribute to the metastasis of HCC cells, aberrant expression of which results in a worse prognosis. Therefore, there is a need to determine the molecular mechanisms underlying metastasis of HCC.

miRNAs may bind to the 3'UTR of target genes and inhibit expression via post-transcriptional regulation (7). A number of studies have demonstrated that miRNAs participate in the occurrence, progression and metastasis of tumors, including gastric cancer and colorectal cancer $(28,29)$. miRNAs are also involved in the pathogenesis and progression of HCC. Hu et al (24) reported that miR-665 promotes $\mathrm{HCC}$ cell migration, invasion and proliferation by decreasing Hippo signaling by targeting protein tyrosine phosphatase receptor type B. Wang et al (30) demonstrated that downregulation of circDYNC1H1 is associated with inhibitory effects on cell proliferation and migration in HCC via miR-140-5p. Yu et al (31) demonstrated that miR-501 acts as an independent prognostic factor which promoted EMT via targeting Jun dimerization protein 2 in HCC. However, the functions of miR-939-3p in tumors have not been extensively studied in HCC to the best of our knowledge. The present study demonstrated that the expression levels of miR-939-3p were increased in HCC tissues and HCC cell lines compared with paired normal tissues and normal cell lines, respectively. Furthermore, it was demonstrated that low expression levels of miR-939-3p were correlated with a more favorable prognosis. Inhibition of miR-939-3p decreased the metastatic ability of HCCLM3 cells and western blotting revealed that miR-939-3p may promote EMT via upregulation of MMP2, MMP9 and vimentin.

An increasing number of studies have demonstrated that ESR1 may act as a tumor suppressor in various cancer types $(16,19)$. The present study demonstrated that the expression levels of ESR1 are downregulated in HCC cell lines compared with normal liver cells. It was demonstrated that inhibition of ESR1 decreased the metastatic ability of HCCLM3 cells, therefore ESR1 inhibition was associated with a more favorable prognosis. Finally, through a dual-luciferase report assay and western blotting, a direct binding association was identified between miR-939-3p and ESR1. miR-939-3p may influence EMT via ESR1, although the present study did not directly show this. Limitations of the present study include the fact that rescue experiments were not performed therefore in further studies these experiments should be performed.

In conclusion, the present study demonstrated that miR-939-3p serves a role in HCC cell invasion and metastasis. miR-939-3p negatively regulated ESR1 by binding to its 3'UTR, therefore the miR-939-3p/ESR1 axis may present a potential target for treatment of $\mathrm{HCC}$ in the future.

\section{Acknowledgements}

Not applicable.

\section{Funding}

The present study was funded by the Medicine and Health Research Foundation of Zhejiang Province (grant no. 2017KY018) and the Zhejiang Provincial Natural Science Foundation of China (grant no. LY18H160043).

\section{Availability of data and materials}

The datasets used and/or analyzed during the present study are available from the corresponding author on reasonable request. 


\section{Authors' contributions}

JX designed the study. FC, XYN, LXC and XYW performed the experiments and analyzed the data. FC and XYN wrote the manuscript. XYN and XYW revised the manuscript.

\section{Ethics approval and consent to participate}

The present study was approved by The Institutional Review Board of the Zhejiang Provincial Peoples' Hospital (Taizhou, China). All patients gave written informed consent to participate in the study and the data were anonymized.

\section{Patient consent for publication}

Not applicable.

\section{Competing interests}

The authors declare that they have no competing interests.

\section{References}

1. Bray F, Ferlay J, Soerjomataram I, Siegel RL, Torre LA and Jemal A: Global cancer statistics 2018: GLOBOCAN estimates of incidence and mortality worldwide for 36 cancers in 185 countries. CA Cancer J Clin 68: 394-424, 2018.

2. Tan A, Yeh SH, Liu CJ, Cheung C and Chen PJ: Viral hepatocarcinogenesis: From infection to cancer. Liver Int 28: 175-188, 2008.

3. Ren FH, Yang H, He RQ, Lu JN, Lin XG, Liang HW, Dang YW, Feng ZB, Chen G and Luo DZ: Analysis of microarrays of miR-34a and its identification of prospective target gene signature in hepatocellular carcinoma. BMC Cancer 18: 12, 2018.

4. Brondfield MN, Dodge JL, Hirose R, Heimbach J, Yao FY and Mehta N: Hepatocellular carcinoma (HCC) patients listed in short wait regions remain advantaged for liver transplant (LT) following 2015 HCC policy change. Liver Transpl: Dec 13, 2019 doi: 10.1002/lt.25701 (Epub ahead of print).

5. Bakheet AMH, Zhao C, Chen JN, Zhang JY, Huang JT, Du Y, Gong LP, Bi YH and Shao CK: Improving pathological early diagnosis and differential biomarker value for hepatocellular carcinoma via RNAscope technology. Hepatol Int: Dec 12, 2019 doi: 10.1007/s12072-019-10006-z (Epub ahead of print).

6. Hu J, Wang E, Liu L, Wang Q, Xia D, Bai W, Tie J, Li X, Yuan J, Yang S, et al: Sorafenib may enhance antitumour efficacy in hepatocellular carcinoma patients by modulating the proportions and functions of natural killer cells. Invest New Drugs: Dec 13, 2019 doi: 10.1007/s10637-019-00885-2 (Epub ahead of print).

7. Berezikov E, Guryev V, van de Belt J, Wienholds E, Plasterk RH and Cuppen E: Phylogenetic shadowing and computational identification of human microRNA genes. Cell 120: 21-24, 2005.

8. Rana TM: Illuminating the silence: Understanding the structure and function of small RNAs. Nat Rev Mol Cell Biol 8: 23-36, 2007.

9. Loosen SH, Schueller F, Trautwein C, Roy S and Roderburg C: Role of circulating microRNAs in liver diseases. World J Hepatol 9: 586-594, 2017.

10. Huang X, Wang L, Liu W and Li F: MicroRNA-497-5p inhibits proliferation and invasion of non-small cell lung cancer by regulating FGF2. Oncol Lett 17: 3425-3431, 2019.

11. Ni JS, Zheng H, Huang ZP, Hong YG, Ou YL, Tao YP, Wang MC, Wang ZG, Yang Y and Zhou WP: MicroRNA-197-3p acts as a prognostic marker and inhibits cell invasion in hepatocellular carcinoma. Oncol Lett 17: 2317-2327, 2019.

12. Tang Z, Fang Y and Du R: MicroRNA-107 induces cell cycle arrests by directly targeting cyclin E1 in ovarian cancer. Biochem Biophys Res Commun 512: 331-337, 2019.

13. Wang J, Chu Y, Xu M, Zhang X, Zhou Y and Xu M: miR-21 promotes cell migration and invasion of hepatocellular carcinoma by targeting KLF5. Oncol Lett 17: 2221-2227, 2019.
14. Chen A,Liu S,Lu X, Wei L and Chen Y: Inhibition of microRNA939 suppresses the development of human nonsmall cell lung cancer via the upregulation of tissue inhibitor of metalloproteinases 2 . Mol Med Rep 18: 4831-4838, 2018.

15. Bjornstrom L and Sjoberg M: Mechanisms of estrogen receptor signaling: Convergence of genomic and nongenomic actions on target genes. Mol Endocrinol 19: 833-842, 2005.

16. Yang J, AlTahan A, Jones DT, Buffa FM, Bridges E, Interiano RB, Qu C, Vogt N, Li JL, Baban D, et al: Estrogen receptor-alpha directly regulates the hypoxia-inducible factor 1 pathway associated with antiestrogen response in breast cancer, Proc Natl Acad Sci USA 112: 15172-15177, 2015.

17. Andruska ND, Zheng X, Yang X, MaoC, Cherian MM, Mahapatra L, Helferich WG and Shapiro DJ: Estrogen receptor $\alpha$ inhibitor activates the unfolded protein response, blocks protein synthesis, and induces tumor regression. Proc Natl Acad Sci USA 112: 4737-4742, 2015.

18. Hishida M, Nomoto S, Inokawa Y, Hayashi M, Kanda M, Okamura Y, Nishikawa Y, Tanaka C, Kobayashi D, Yamada S, et al: Estrogen receptor 1 gene as a tumor suppressor gene in hepatocellular carcinoma detected by triple-combination array analysis. Int J Oncol 43: 88-94, 2013.

19. Tu CC, Kumar VB, Day CH, Kuo WW, Yeh SP, Chen RJ, Liao CR, Chen HY, Tsai FJ, Wu WJ and Huang CY: Estrogen receptor alpha (ESR1) over-expression mediated apoptosis in Hep3B cells by binding with SP1 proteins. J Mol Endocrinol 51: 203-212, 2013.

20. Nagy A, Lánczky A, Menyhárt O and Győrffy B: Validation of miRNA prognostic power in hepatocellular carcinoma using expression data of independent datasets. Sci Rep 8: 9227, 2018.

21. Wan J, Liu H, Yang L, Ma L, Liu J and Ming L: JMJD6 promotes hepatocellular carcinoma carcinogenesis by targeting CDK4. Int J Cancer 144: 2489-2500, 2019.

22. Hu D, Hu Y, Xu W, Yu H, Yang N, Ni S and Fu R: miR203 inhibits the expression of collagenrelated genes and the proliferation of hepatic stellate cells through a SMAD3dependent mechanism. Mol Med Rep 16: 1248-1254, 2017.

23. Wan X, Cheng C, Shao Q, Lin Z, Lu S and Chen Y: CD24 promotes HCC progression via triggering Notch-related EMT and modulation of tumor microenvironment. Tumour Biol 37: 6073-6084, 2016.

24. Hu Y, Yang C, Yang S, Cheng F, Rao J and Wang X: miR-665 promotes hepatocellular carcinoma cell migration, invasion, and proliferation by decreasing Hippo signaling through targeting PTPRB. Cell Death Dis 9: 954, 2018.

25. Zhang XP, Jiang YB, Zhong CQ, Ma N, Zhang EB, Zhang F, Li JJ, Deng YZ, Wang K, Xie D and Cheng SQ: PRMT1 promoted HCC growth and metastasis in vitro and in vivo via activating the STAT3 signalling pathway. Cell Physiol Biochem 47: 1643-1654, 2018.

26. Yu H, Shen H, Zhang Y, Zhong F, Liu Y, Qin L and Yang P: CAV1 promotes HCC cell progression and metastasis through Wnt/ $\beta$-catenin pathway. PLoS One 9: e106451, 2014.

27. Li Y, Chen L, Chan TH, Liu M, Kong KL, Qiu JL, Li Y, Yuan YF and Guan XY: SPOCK1 is regulated by CHD1L and blocks apoptosis and promotes HCC cell invasiveness and metastasis in mice. Gastroenterology 144: 179-191 e174, 2013.

28. Feng L, Jing L, Han J, Wang G, Liu Y, Zhang X, Wang Y, Wang F, Ma H and Liu Y: MicroRNA 486-3p directly targets BIK and regulates apoptosis and invasion in colorectal cancer cells. Onco Targets Ther 11: 8791-8801, 2018.

29. Liang M, Shi B, Liu J, He L, Yi G, Zhou L, Yu G and Zhou X: Downregulation of miR203 induces overexpression of PIK3CA and predicts poor prognosis of gastric cancer patients. Drug Des Devel Ther 9: 3607-3616, 2015.

30. Wang ZY, Zhu Z, Wang HF, Qin B, Liu J, Yao XH, Li WC and Chen KS: Downregulation of circDYNC1H1 exhibits inhibitor effect on cell proliferation and migration in hepatocellular carcinoma through miR-140-5p. J Cell Physiol 234: 17775-17785, 2019.

31. Yu W, Deng W, Zhao Q, Zhuang H, Zhang C and Jian Z: miR-501 acts as an independent prognostic factor that promotes the epithelial-mesenchymal transition through targeting JDP2 in hepatocellular carcinoma. Hum Cell 32: 343-351, 2019.

This work is licensed under a Creative Commons Attribution-NonCommercial-NoDerivatives 4.0 International (CC BY-NC-ND 4.0) License. 